\title{
In This Issue: October 2020
}

\author{
Adam M. Brenner ${ }^{1}$ \\ Published online: 15 September 2020 \\ (C) Academic Psychiatry 2020
}

The October 2020 issue of Academic Psychiatry will appear as we begin another application and recruitment season for general psychiatry residency programs. Due to the COVID-19 pandemic, programs are preparing for a virtual environment, and students are worrying about how this unprecedented format will affect their prospects. In this context, some of the perennial stresses of the match process will be felt even more acutely. Readers involved in the residency application process will probably have already noticed a growing anxiety among applicants in recent years about the match. It is even more important then that we pay attention to those students who do not receive a spot in the initial match or Supplemental Offer and Acceptance Program (SOAP) process. Bailey et al. [1] report on a 10-year review of a program designed to help unmatched students, and the accompanying editorial by Balon et al. [2] discusses the resources available to these students, calls on academic departments to develop programs on their behalf, and considers how to best provide guidance to the individual.

Another question is whether to disclose a history of psychiatric illness in an application to psychiatry residency [3]. This decision has always been a very thorny one, and Pheister et al.'s report "The Impact of Mental Illness Disclosure in Applying for Residency" [4] is a valuable contribution to our literature. Unfortunately, their data suggest that applicants may still pay more of a price for disclosing a psychiatric illness compared with a medical illness. The study was not powered to answer the question of whether this bias and its consequences may be less in psychiatry programs than in other specialties. Aggarwal et al. [5] discuss the implications of this study and others for the practical advice we might offer to applicants.

The topic of mental health treatment—and its stigma - for students and health care professionals is also addressed in

Adam M. Brenner

adam.brenner@utsouthwestern.edu

1 University of Texas Southwestern Medical Center, Dallas, TX, USA
Goldenberg and Wilkins' [6] thoughtful reflection on the challenges to privacy and boundaries when a medical student becomes an inpatient on a teaching service. Goldberg et al. [7] also address some of the complex dynamics of treating a fellow professional, but they shift the focus to the realm of providing outpatient therapy for other therapists. We in academic psychiatry are often aware of the need to maintain a boundary, lest psychotherapy supervision shade into psychotherapy treatment of the trainee. But there is also a parallel tensionhow to help a patient who is a therapist and whose material inevitably includes accounts of their work with their patients, without slipping into a posture of supervision. Because the tasks of managing the parallel developments of our professional and personal selves are inevitably intertwined, this job is complex and subtle!

Although these papers offer guidance for academic psychiatrists when we are in the "treater" position, they may have the greatest benefit in helping break down the barriers to seeing ourselves in the "patient" role. Goldberg et al. [7] describe this situation as the "us vs. them" sense - the wish to manage our anxiety by dividing people into the categories of those who suffer mentally and those who help them. Many of us know from direct and compelling experience how false this dichotomy is.

The current residency application season also begins as we are engaged in a re-examination of diversity within our profession in general, within academic psychiatry, and among psychiatry trainees. The empirical report "Diversity by Race, Ethnicity, and Sex within the US Psychiatry Physician Workforce" [8] collates and compares multiple sources of publicly available data. Wyse et al. [9] provide both a snapshot of where we are today and a window into the question, Are we making progress? It is good to see that prior efforts to widen the pipeline of underrepresented minority (URM) applicants into psychiatry have yielded some improvements - percentages of URM psychiatry residents are better than several decades ago and are better for psychiatry residents than for all medical school graduates. And yet, these numbers remain dismally low. Black residents comprise 
Table 1 Academic Psychiatry calls for papers

\begin{tabular}{|c|c|c|}
\hline Topic & Content & Due Date \\
\hline Diversity, Equity, and Inclusion & $\begin{array}{l}\text { Diversity, equity, and inclusion represent an increasingly vital topic } \\
\text { in academic psychiatry, encompassing faculty and trainee recruitment } \\
\text { and retention, how and what to teach, mentorship and supervision, } \\
\text { faculty development, health care access and disparities, social } \\
\text { determinants of mental health, cultural humility and competency, } \\
\text { and more. "Diversity" includes race, ethnicity, culture, nationality, } \\
\text { religion, language, sexual orientation, gender identity, age, experiences, } \\
\text { political party affiliation, and physical abilities; some of these are being } \\
\text { importantly re-conceptualized in current discourse }\end{array}$ & October 1,2020 \\
\hline COVID-19 and Psychiatry Education & $\begin{array}{l}\text { We are looking for papers that have data about impact, interventions, and so } \\
\text { forth. We are interested both in how programs have responded to the acute } \\
\text { crisis (e.g., impact on technology use) and in how programs are teaching } \\
\text { about the different intersections of COVID-19 and mental health } \\
\text { (individual and population). We are also interested in papers that address } \\
\text { problems or barriers encountered and resources that may be useful for } \\
\text { academic psychiatrists, particularly if such papers include a foundation } \\
\text { for assessment of efficacy and means of replicating }\end{array}$ & May 20, 2021 \\
\hline
\end{tabular}

All papers undergo review, and future publication is not guaranteed. See the journal's instructions for authors for more details [9].

$7.1 \%$ of the total, compared with $13.3 \%$ of the US population, and Hispanic residents comprise $8.9 \%$, compared with $17.8 \%$. We have a lot of work left to do.

For those of us in academic settings, perhaps the most alarming finding is the very modest gains, or no gains at all, of the percentage of URM among psychiatry faculty. From 1987 to 2016, Hispanics moved from 3.2 to $5.2 \%$ of faculty, while Blacks made no gains whatsoever. The percentage of Black psychiatry faculty has remained at essentially $3 \%$ throughout these decades, to the present. Beyond knowing the demographics, we need to understand the cause behind these stagnant numbers. We in academic psychiatry leadership need to be asking difficult questions in self-reflection, and we need to find ways to support empirical research that both defines the barriers and tests interventions to overcome them. We have to do better.

We hope that you find the October 2020 issue of Academic Psychiatry helpful as you grapple with this array of challenges and opportunities. Please feel free to contact us with any feedback. The journal also has two open calls for papers (Table 1): "Diversity, Equity, and Inclusion" and "COVID-19 and Psychiatry Education" [9]. We hope you will consider Academic Psychiatry as a place of publication for your work.

\section{Compliance with Ethical Standards}

Conflict of Interest The author declares that he has no conflicts of interest.

\section{References}

1. Bailey RK, Saldana AM, Mohayed MO, et al. Ten-year review of the extern program: a solution to an unsuccessful match? Acad Psychiatry. 2020. https://doi.org/10.1007/s40596-020-01246-5.

2. Balon R, Morreale MK, Coverdale J, et al. Medical students who do not match to psychiatry: what should they do, and what should we do? Acad Psychiatry. 2020. https://doi.org/10.1007/s40596-02001295-w.

3. Brenner AM, Balon R, Guerrero APS, et al. Training as a psychiatrist when having a psychiatric illness. Acad Psychiatry. 2018;42:592-7.

4. Pheister M, Peters RM, Wrzosek MI. The impact of mental illness disclosure in applying for residency. Acad Psychiatry. 2020. https:// doi.org/10.1007/s40596-020-01227-8.

5. Aggarwal R, Coverdale J, Balon R, et al. To disclose or not: residency application and psychiatric illness. Acad Psychiatry. 2020. https:// doi.org/10.1007/s40596-020-01296-9.

6. Goldenberg MN, Wilkins KM. A medical student is psychiatrically hospitalized. Acad Psychiatry. 2019. https://doi.org/10.1007/ s40596-019-01172-1.

7. Goldberg M, Stanger Elran R, Mayer Y, Lurie I. The treatment of a patient who is also a mental health practitioner: special considerations and recommendations. Acad Psychiatry. 2020. https://doi. org/10.1007/s40596-020-01280-3.

8. Wyse R, Hwang W, Ahmed AA, et al. Diversity by race, ethnicity, and sex within the US psychiatry physician workforce. Acad Psychiatry. 2020. https://doi.org/10.1007/s40596-020-01276-z.

9. Academic Psychiatry. Instructions for Authors. July 2020. Available at https://www.springer.com/journal/40596/submission-guidelines. Accessed 28 Aug 2020.

Publisher's Note Springer Nature remains neutral with regard to jurisdictional claims in published maps and institutional affiliations. 